\title{
Kaon Decay into Three Photons Revisited
}

\author{
Shu-Yu Ho* \\ Department of Physics, California Institute of Technology \\ Pasadena, CA 91125, USA \\ E-mail: sho3ecaltech.edu \\ Jusak Tandean ${ }^{\dagger}$ \\ Department of Physics and Center for Theoretical Sciences, National Taiwan University \\ Taipei 106, Taiwan \\ E-mail: jtandean@yahoo.com
}

We take another look at the rare kaon decay into three photons. Specifically, after imposing the requirements of gauge invariance and Bose symmetry, we derive a general form of the decay amplitude, including both parity-conserving and parity-violating contributions. Subsequently, we adopt a chiral-Lagrangian approach in conjunction with dimensional analysis arguments to estimate the branching ratios of $K_{\mathrm{L}, \mathrm{S}} \rightarrow 3 \gamma$ in the standard model, obtaining values as large as $\mathscr{B}\left(K_{\mathrm{L}} \rightarrow 3 \gamma\right) \simeq 7 \times 10^{-17}$ and $\mathscr{B}\left(K_{\mathrm{S}} \rightarrow 3 \gamma\right) \simeq 1 \times 10^{-19}$, which exceed those found previously by a few orders of magnitude. Measurements of $\mathscr{B}\left(K_{\mathrm{L}, \mathrm{S}} \rightarrow 3 \gamma\right)$ substantially bigger than these numbers would likely hint at the presence of new physics beyond the standard model.

Flavor Physics \& CP Violation 2015

May 25-29, 2015

Nagoya, Japan

\footnotetext{
* Speaker.

$\dagger$ This research was supported in part by the National Science Council and National Center for Theoretical Sciences of Taiwan.
} 
The kaon decays into three photons, $K_{\mathrm{L}} \rightarrow 3 \gamma$ and $K_{\mathrm{S}} \rightarrow 3 \gamma$, can happen in the absence of $C P$ violation. Based on the experimental branching ratio $\mathscr{B}\left(K_{\mathrm{L}} \rightarrow 2 \gamma\right) \simeq 5.5 \times 10^{-4}$ [1], one might then naively expect that $\mathscr{B}\left(K_{\mathrm{L}} \rightarrow 3 \gamma\right) \sim \alpha_{\text {em }} \mathscr{B}\left(K_{\mathrm{L}} \rightarrow 2 \gamma\right) \sim 4 \times 10^{-6}$. However, this is already way higher than the existing measured limit $\mathscr{B}\left(K_{\mathrm{L}} \rightarrow 3 \gamma\right)<7.4 \times 10^{-8}[1,2]$. As for its $K_{\mathrm{S}}$ counterpart, there is currently no empirical information available about it, but its rate is likely to be more suppressed than expected as well.

The considerable smallness of the $K \rightarrow 3 \gamma$ rate turns out to stem from the conditions imposed on the decay amplitude by gauge invariance and Bose symmetry [3]. Gauge invariance compels the total angular momentum $J$ of any two photons in the $3 \gamma$ final-state to be nonzero, while Bose statistics disallows the $\gamma \gamma$ pair having $J=1$. Since each pair of the photons has $J \geq 2$, the amplitude suffers from a sizable number of angular-momentum suppression factors.

The $K_{\mathrm{L}, \mathrm{S}} \rightarrow 3 \gamma$ rates were first estimated over 2 decades ago in [3], using a simple model in which $K \rightarrow 3 \gamma$ proceeds from $K \rightarrow \pi^{0} \pi^{0} \gamma$ with $\pi^{0} \pi^{0}$ immediately converting into $\gamma \gamma$. This led to $\mathscr{B}\left(K_{\mathrm{L}} \rightarrow 3 \gamma\right) \sim 3 \times 10^{-19}$ and $\mathscr{B}\left(K_{\mathrm{S}} \rightarrow 3 \gamma\right) \sim 5 \times 10^{-22}$ [3]. As this rough determination relied on only 1 diagram, possibly other contributions exist that can enhance the rates. Here we present the results of a more recent study [4] revisiting these decays and attaining much higher numbers.

The $K \rightarrow 3 \gamma$ amplitude generally consists of two terms describing the parity conserving (PC) and parity violating (PV) components of the transition, namely

$$
\mathscr{M}(K \rightarrow 3 \gamma)=\mathscr{M}_{\mathrm{PC}}^{K}+\mathscr{M}_{\mathrm{PV}}^{K}, \quad \mathscr{M}_{\mathrm{PC}}^{K}=\varepsilon_{1 \alpha}^{*} \varepsilon_{2 \eta}^{*} \varepsilon_{3 \mu}^{*} M_{\mathrm{PC}}^{\alpha \eta \mu}, \quad \mathscr{M}_{\mathrm{PV}}^{K}=\varepsilon_{1 \alpha}^{*} \varepsilon_{2 \eta}^{*} \varepsilon_{3 \mu}^{*} M_{\mathrm{PV}}^{\alpha \eta \mu},
$$

where $\varepsilon_{1,2,3}$ are the photon polarization vectors. Each of $\mathscr{M}_{\mathrm{PV}, \mathrm{PC}}^{K}$ has to respect gauge invariance and be symmetric under interchange of any two of the photons. As discussed in detail in [4], imposing these requirements with on-shell photons, after some algebra we arrive at ${ }^{1}$

$$
\begin{aligned}
M_{\mathrm{PV}}^{\alpha \eta \mu}= & {\left[g^{\alpha \eta}\left(k_{1}^{\mu} y-k_{2}^{\mu} x\right)+g^{\eta \mu}\left(k_{2}^{\alpha} x-k_{3}^{\alpha} z\right)+g^{\alpha \mu}\left(k_{3}^{\eta} z-k_{1}^{\eta} y\right)+k_{3}^{\alpha} k_{1}^{\eta} k_{2}^{\mu}-k_{2}^{\alpha} k_{3}^{\eta} k_{1}^{\mu}\right] G(x, y, z) } \\
& +\left(g^{\alpha \eta} z-k_{2}^{\alpha} k_{1}^{\eta}\right)\left(k_{1}^{\mu} y-k_{2}^{\mu} x\right) F(x, y, z)+\left(g^{\eta \mu} y-k_{3}^{\eta} k_{2}^{\mu}\right)\left(k_{2}^{\alpha} x-k_{3}^{\alpha} z\right) F(z, x, y) \\
& +\left(g^{\alpha \mu} x-k_{3}^{\alpha} k_{1}^{\mu}\right)\left(k_{3}^{\eta} z-k_{1}^{\eta} y\right) F(y, z, x), \\
M_{\mathrm{PC}}^{\alpha \eta \mu}= & \left(g^{\alpha \eta} \varepsilon^{\mu \rho \sigma \tau}+g^{\rho \sigma} \varepsilon^{\alpha \eta \mu \tau}+g^{\eta \rho} \varepsilon^{\alpha \mu \sigma \tau}-g^{\alpha \sigma} \varepsilon^{\eta \mu \rho \tau}+g^{\eta \mu} \varepsilon^{\alpha \rho \sigma \tau}+g^{\sigma \tau} \varepsilon^{\alpha \eta \mu \rho}+g^{\mu \sigma} \varepsilon^{\alpha \eta \rho \tau}\right. \\
& \left.-g^{\eta \tau} \varepsilon^{\alpha \mu \rho \sigma}+g^{\alpha \mu} \varepsilon^{\eta \rho \sigma \tau}+g^{\rho \tau} \varepsilon^{\alpha \eta \mu \sigma}+g^{\mu \rho} \varepsilon^{\alpha \eta \sigma \tau}-g^{\alpha \tau} \varepsilon^{\eta \mu \rho \sigma}\right) k_{1 \rho} k_{2 \sigma} k_{3 \tau} \mathscr{G}(x, y, z) / 3 \\
+ & {\left[\left(g^{\alpha \eta} z-k_{2}^{\alpha} k_{1}^{\eta}\right) \varepsilon^{\mu \rho \sigma \tau} \mathscr{F}(x, y, z)+\left(g^{\eta \mu} y-k_{3}^{\eta} k_{2}^{\mu}\right) \varepsilon^{\alpha \rho \sigma \tau} \mathscr{F}(z, x, y)\right.} \\
& \left.+\left(g^{\alpha \mu} x-k_{3}^{\alpha} k_{1}^{\mu}\right) \varepsilon^{\eta \rho \sigma \tau} \mathscr{F}(y, z, x)\right] k_{1 \rho} k_{2 \sigma} k_{3 \tau} \\
+ & {\left[\left(k_{2}^{\mu} k_{1}^{\tau}-k_{1}^{\mu} k_{2}^{\tau}\right) \varepsilon^{\alpha \eta \rho \sigma} \mathscr{H}(x, y, z)+\left(k_{3}^{\alpha} k_{2}^{\rho}-k_{2}^{\alpha} k_{3}^{\rho}\right) \varepsilon^{\eta \mu \sigma \tau} \mathscr{H}(z, x, y)\right.} \\
& \left.+\left(k_{1}^{\eta} k_{3}^{\sigma}-k_{3}^{\eta} k_{1}^{\sigma}\right) \varepsilon^{\alpha \mu \rho \tau} \mathscr{H}(y, z, x)\right] k_{1 \rho} k_{2 \sigma} k_{3 \tau},
\end{aligned}
$$

where $k_{1,2,3}$ are the photon momenta, $x=k_{1} \cdot k_{3}, y=k_{2} \cdot k_{3}, z=k_{1} \cdot k_{2}$, and the functions $F, G$, $\mathscr{F}, \mathscr{G}$, and $\mathscr{H}$ must be free of kinematic singularities and satisfy the relations

$$
\begin{aligned}
F(u, v, w) & =-F(v, u, w), \quad G(u, v, w)=-G(v, u, w)=-G(w, v, u)=-G(u, w, v), \\
\mathscr{F}(u, v, w) & =-\mathscr{F}(v, u, w), \quad \mathscr{H}(u, v, w)=-\mathscr{H}(v, u, w), \\
\mathscr{G}(u, v, w) & =-\mathscr{G}(v, u, w)=-\mathscr{G}(w, v, u)=-\mathscr{G}(u, w, v) .
\end{aligned}
$$

with $u, v, w$ each being any one of the invariants $k_{i} \cdot k_{j}$.

\footnotetext{
${ }^{1}$ We derived $M_{\mathrm{PC}}$ in (3) with the aid of Schouten's identity, more examples of which can be found in [5].
} 
In the sum of $\left|\mathscr{M}_{\mathrm{PV}}^{K}+\mathscr{M}_{\mathrm{PC}}^{K}\right|^{2}$ over the photon polarizations, the interference between $\mathscr{M}_{\mathrm{PV}, \mathrm{PC}}^{K}$ vanishes. The corresponding decay rate is given by

$$
\begin{aligned}
& \Gamma(K \rightarrow 3 \gamma)=\frac{1}{256 \pi^{3} m_{K}^{3}} \frac{1}{3 !} \int d s_{12} d s_{23} \sum_{\mathrm{pol}}\left(\left|\mathscr{M}_{\mathrm{PV}}^{K}\right|^{2}+\left|\mathscr{M}_{\mathrm{PC}}^{K}\right|^{2}\right), \\
& \sum_{\text {pol }}\left|\mathscr{M}_{\mathrm{PV}}^{K}\right|^{2}=4\left\{\left|F_{1}\right|^{2} z^{2}+\left|F_{2}\right|^{2} y^{2}+\left|F_{3}\right|^{2} x^{2}+2|G(x, y, z)|^{2}\right. \\
& \left.+\operatorname{Re}\left[F_{1}^{*} F_{2} y z+F_{2}^{*} F_{3} x y+F_{3}^{*} F_{1} x z+2\left(F_{1}^{*} z+F_{2}^{*} y+F_{3}^{*} x\right) G(x, y, z)\right]\right\} x y z, \\
& \sum_{\mathrm{pol}}\left|\mathscr{M}_{\mathrm{PC}}^{K}\right|^{2}=4\left\{\left(\left|\mathscr{F}_{1}\right|^{2}+\left|\mathscr{H}_{1}\right|^{2}\right) z^{2}+\left(\left|\mathscr{F}_{2}\right|^{2}+\left|\mathscr{H}_{2}\right|^{2}\right) y^{2}+\left(\left|\mathscr{F}_{3}\right|^{2}+\left|\mathscr{H}_{3}\right|^{2}\right) x^{2}+2 \mid \mathscr{G}_{\left.(x, y, z)\right|^{2}}\right. \\
& +\operatorname{Re}\left[\left(\mathscr{F}_{1}^{*}+\mathscr{H}_{1}^{*}\right)\left(\mathscr{F}_{2}+\mathscr{H}_{2}+2 \mathscr{G}(x, y, z) / y\right) y z\right. \\
& +\left(\mathscr{F}_{2}^{*}+\mathscr{H}_{2}^{*}\right)\left(\mathscr{F}_{3}+\mathscr{H}_{3}+2 \mathscr{G}(x, y, z) / x\right) x y \\
& \left.\left.+\left(\mathscr{F}_{3}^{*}+\mathscr{H}_{3}^{*}\right)\left(\mathscr{F}_{1}+\mathscr{H}_{1}+2 \mathscr{G}(x, y, z) / z\right) x z\right]\right\} x y z,
\end{aligned}
$$

where the 3 ! accounts for the 3 photons being identical particles, $s_{m n}=\left(k_{m}+k_{n}\right)^{2}, F_{1}=F(x, y, z)$, $F_{2}=F(z, x, y), F_{3}=F(y, z, x)$, and similarly for $\mathscr{F}_{1,2,3}$ and $\mathscr{H}_{1,2,3}$. We note that the preceding formulas apply more generally to any other neutral pseudoscalar particle decaying into $3 \gamma$, and they also work for the decay of a neutral scalar particle if the PC and PV parts are interchanged.

To explore the leading contributions, we adopt a chiral-Lagrangian approach [6]. Accordingly, they are expected to arise from the relevant portions in the chiral expansion and yield terms in the functions $F, G, \mathscr{F}, \mathscr{G}$, and $\mathscr{H}$ with the lowest numbers of powers of the photon momenta $k_{i}$. Since there are in principle many contributions to the amplitude, from tree and loop diagrams, with unknown parameters, it suffices to consider just one representative and rely on dimensional-analysis arguments to evaluate its size.

Treating $K_{\mathrm{L}} \rightarrow 3 \gamma$ first and ignoring $C P$ violation, we can focus on $\mathscr{M}_{\mathrm{PV}}^{K}$. From the simplest formulas $F(u, v, w)=c_{F}(u-v)$ and $G(u, v, w)=c_{G}[(u-v) f(w)+(v-w) f(u)+(w-u) f(v)]$ fulfilling (4), with $c_{F, G}$ being constants and $f$ any well-behaved function, we see that $F$ and $G$ contain at least 2 and 4 powers of $k_{i}$, respectively. Thus $M_{\mathrm{PV}}$ involves at least 7 powers of $k_{i}$.

To assess the leading contributions to $M_{\mathrm{PV}}$, we look at a weak chiral Lagrangian for standardmodel strangeness-changing, $|\Delta S|=1$, transitions which is parity odd, has 7 derivatives, and couples $K$ to $3 \gamma$ in a gauge-invariant way. As is well known, such a chiral Lagrangian proceeds from the dominant left-handed chiral octet piece of the weak interactions of light quarks [6] and has to be invariant under the $C P$ transformation combined with the switching of $s$ and $d$ quarks [7]. An example with the desired properties is

$$
\begin{aligned}
\mathscr{L}_{\mathrm{PV}} & =c_{7}\left\langle\xi^{\dagger} h \xi\left(\nabla^{\alpha} \mathscr{V}^{\mu v}\right)\left[\mathscr{U}^{\rho} \nabla_{\alpha} \mathscr{V}_{\rho \sigma}+\left(\nabla_{\sigma} \mathscr{V}_{\rho \alpha}\right) \mathscr{U}^{\rho}\right] \nabla^{\sigma} \mathscr{V}_{\mu v}\right\rangle+\text { H.c. } \\
& =\frac{8 \sqrt{2} c_{7} e^{3}}{27 f_{\pi}} \partial^{\alpha} F^{\mu v}\left(\partial_{\alpha} F_{\rho \sigma}+\partial_{\sigma} F_{\rho \alpha}\right) \partial^{\rho} \bar{K}^{0} \partial^{\sigma} F_{\mu v}+\cdots+\text { H.c. },
\end{aligned}
$$

where $c_{7}$ is a constant, $F_{\mu v}=\partial_{\mu} A_{v}-\partial_{v} A_{\mu}$ is the usual photon field strength tensor, and other details can be found in [4]. This translates into

$$
F(u, v, w)=\frac{32 \sqrt{2} i c_{7} e^{3}}{27 f_{\pi}}(u-v), \quad G(u, v, w)=0 .
$$

Assuming $C P$ conservation and adopting the convention $K_{\mathrm{L}}=\left(K^{0}+\bar{K}^{0}\right) / \sqrt{2}$, we then obtain

$$
\sum_{\text {pol }}\left|\mathscr{M}\left(K_{\mathrm{L}} \rightarrow 3 \gamma\right)\right|^{2}=\frac{\left|128 c_{7}\right|^{2} e^{6}}{729 f_{\pi}^{2}}\left(x^{2} y^{2}+y^{2} z^{2}+x^{2} z^{2}-x y z^{2}-x y^{2} z-x^{2} y z\right) x y z .
$$


Since it is not yet possible to compute $c_{7}$ rigorously from the quark-level parameters, we estimate it with the aid of naive dimensional analysis [8]. Thus we get the order-of-magnitude value

$$
c_{7} \sim \frac{G_{\mathrm{F}} \lambda_{\mathrm{C}} f_{\pi}^{4}}{\sqrt{2} \Lambda^{8}} \simeq 1.0 \times 10^{-9} \mathrm{GeV}^{-6},
$$

where $\lambda_{\mathrm{C}}=0.22$ is the Cabibbo mixing parameter and $\Lambda$ represents the scale at which the chiral Lagrangian approach breaks down, which suggests we set $\Lambda=m_{\rho}=775 \mathrm{MeV}$ [1]. The resulting branching ratio is $\mathscr{B}\left(K_{\mathrm{L}} \rightarrow 3 \gamma\right) \sim 7.4 \times 10^{-17}$.

As for $K_{\mathrm{S}} \rightarrow 3 \gamma$, the amplitude is dominated by $\mathscr{M}_{\mathrm{PC}}^{K}$, and we can pick the leading-order form $\mathscr{F}(u, v, w) \sim \mathscr{H}(u, v, w)=\tilde{c}(u-v)$ with $\tilde{c}$ being a constant and $\mathscr{G}=0$, satisfying (4). Hence the situation is similar to that of $\mathscr{M}_{\mathrm{PV}}^{K}$ with $F$ and $G$ in (7). More precisely, making a comparison of $\Sigma_{\text {pol }}\left|\mathscr{M}_{\mathrm{PC}}^{K}\right|^{2}$ and $\Sigma_{\text {pol }}\left|\mathscr{M}_{\mathrm{PV}}^{K}\right|^{2}$ above for the two cases, respectively, one can see that their decay distributions have the same functional dependence on $x, y$, and $z$. It follows that $\Gamma\left(K_{\mathrm{S}} \rightarrow 3 \gamma\right)$ can be expected to be roughly of the same order as $\Gamma\left(K_{\mathrm{L}} \rightarrow 3 \gamma\right)$. Interestingly, the measured rates of their $2 \gamma$ counterparts are also of similar order, $\Gamma\left(K_{\mathrm{S}} \rightarrow 2 \gamma\right) \sim 2.7 \Gamma\left(K_{\mathrm{L}} \rightarrow 2 \gamma\right)$ [1]. In view of $\mathscr{B}\left(K_{\mathrm{L}} \rightarrow 3 \gamma\right)$ in the last paragraph, we can therefore predict that $\mathscr{B}\left(K_{\mathrm{S}} \rightarrow 3 \gamma\right) \sim 1 \times 10^{-19}$.

In conclusion, we have revisited the rare kaon decay $K \rightarrow 3 \gamma$, which is expected to be much suppressed because its amplitude has a large number of angular momentum suppression factors. We construct a general form of the amplitude which adheres to the requisites of gauge invariance and Bose symmetry and includes both parity-conserving and parity-violating components. In addition, we provide an expression for the squared amplitude, summed over the photon polarizations, which can be useful to produce a Dalitz plot distribution of the decay. These results are applicable generally to the decay of any spinless particle into $3 \gamma$. More specifically, we explore the leading-order contributions to the amplitudes for $K_{\mathrm{L}, \mathrm{S}} \rightarrow 3 \gamma$ in the standard model by means of a chiral-Lagrangian technique along with dimensional-analysis reasoning. This finally leads us to branching ratios that are bigger by a few orders of magnitude than those calculated before, but still tiny. Nevertheless, any experimental findings on $\mathscr{B}\left(K_{\mathrm{L}, \mathrm{S}} \rightarrow 3 \gamma\right)$ significantly exceeding our predictions would likely signal the effects of new physics.

\section{References}

[1] K.A. Olive et al. [Particle Data Group Collaboration], Chin. Phys. C 38, 090001 (2014).

[2] Y.C. Tung et al. [E391a Collaboration], Phys. Rev. D 83, 031101 (2011).

[3] P. Heiliger, B. McKellar, and L.M. Sehgal, Phys. Lett. B 327, 145 (1994).

[4] S.Y. Ho and J. Tandean, Phys. Rev. D 82, 114010 (2010).

[5] H.W. Fearing and S. Scherer, Phys. Rev. D 53, 315 (1996); A.L. Bondarev, Theor. Math. Phys. 101 (1994) 1376; O. Antipin and G. Valencia, Phys. Rev. D 74, 054015 (2006).

[6] J.F. Donoghue, E. Golowich, and B.R. Holstein, Dynamics of the Standard Model (Cambridge University Press, Cambridge, 1992).

[7] C.W. Bernard, T. Draper, A. Soni, H.D. Politzer, and M.B. Wise, Phys. Rev. D 32, 2343 (1985).

[8] A. Manohar and H. Georgi, Nucl. Phys. B 234, 189 (1984); H. Georgi, Phys. Lett. B 298, 187 (1993). 